\title{
Construcción social de la
}

\section{categoría desplazado,}

vínculos con las instituciones e implicaciones en la política pública

Social Construction of Displaced Individuals as a Category, Their Relationship with Institutions and Public Policy Implications

Construção social da categoria de deslocado, vínculos com as instituições e implicações na política publica

\section{Gina Marcela Arias Rodríguez $\bullet$ Colombia}

\author{
Recibido el 7 de abril de 2011 - aprobado el 21 de mayo de 2011
}

\section{Resumen}

Objetivos. Se analizó desde una perspectiva cualitativa, cómo los distintos actores implicados en el fenómeno del desplazamiento forzado participan en la construcción social de éste como "problema" y cómo se construye socialmente la categoría "desplazado". A su vez, se reflexionó sobre los vínculos que esta población construye con las instituciones gubernamentales y no gubernamentales y qué implicaciones tiene ello para el diseño, formulación y ejecución de las políticas públicas. Materiales y método. dichos análisis fueron realizados desde el enfoque construccionista de la Psicología Social Crítica. El tipo de investigación empleado fue el de la Teoría Fundamentada, realizando muestreo teórico y empleando los mecanismos de comparación y de codificación abierta, axial y selectiva. Resultados. Las personas en situación de desplazamiento reconstituyen su identidad alrededor de la figura del "desplazado", esta categoría les permite definirse y disponerse ante las instituciones y las organizaciones. Las personas entrevistadas han sido objeto de la estigmatización que hace la población receptora sobre ellos como auxiliadores de la guerrilla o como personas que compiten con los pobres por la atención de las instituciones del Estado. Conclusiones. La condición de desplazado enmarca al sujeto como víctima, como vividor, como sujeto de derechos con capacidad de restituir su condición de sujeto a pesar de los hechos violentos y la situación de marginalidad a la que se ve enfrentado con la llegada a un contexto diferente y muchas veces hostil.

Palabras claves: Desplazamiento forzado, construccionismo, víctima, sujeto de derechos, instituciones gubernamentales y no gubernamentales, vínculos.

* Psicóloga • Magíster en Psicología Comunitaria • Docente de la Universidad Católica de Pereira gina_arias82@yahoo.com.ar 


\begin{abstract}
Objectives. This paper aims at showing a qualitative analysis of how different actors involved in the forced displacement phenomenon participate in their social construction as a 'problem' and how the 'displaced individual' category is socially built. It is reflected on the relationship of this population with governmental and non-governmental organizations as well as their implications on public policy design, formulation and implementation. Materials and method. A constructivist approach of the critical social psychology served to carry out the analyses. Grounded theory was the type of study performed by means of a theoretical sampling, considering comparative mechanisms and open, axial and selective codification. Results. Displaced individuals recreate their identity based on the 'displaced' representation. This category allows them to be defined and placed accordingly within institutions and organizations. The interviewed individuals have been stigmatized by the community as guerrilla helpers or as individuals competing with poor for getting the state institutions' attention. Conclusions. Displaced condition places individuals as victims, abusers, individuals of rights, who can be able to restore their condition as individuals, in spite of the violence and social exclusion they face when dealing with a different and in most cases hostile environment.
\end{abstract}

Keywords: Forced displaced phenomenon, constructivism, victim, individual of rights, government and non-governmental organizations, relationships.

\title{
Resumo
}

Objetivos. Analisou se desde uma perspectiva qualitativa, como os distintos atores implicados no fenômeno de deslocamento forçado participam na construção social deste como "problema" e como se constrói socialmente a categoria de "deslocado". Ao mesmo tempo, reflexionou se sobre os vínculos que esta povoação constrói com as instituições governamentais e não governamentais e que implicações têm isto para o desenho, formulação e execução das políticas públicas. Materiais e método. Os analises foram realizados desde o enfoque construtivo da Psicologia Social Critica. O tipo de pesquisa empregado foi da Teoria Fundamentada, realizando uma amostra teórica e empregando os mecanismos de comparação e de codificação aberta, axial e seletiva. Resultados. As pessoas em situação de deslocamento reconstroem sua identidade ao redor da figura do "deslocado", esta categoria permite lhes definir se e se dispor ante as instituições e as organizações. As pessoas entrevistadas tem sido objeto da estigmatizarão que faz a povoação receptora sobre eles como auxiliadores da guerrilha ou como pessoas que permitem com os pobres pela atenção das instituições do Estado. Conclusões. A condição de deslocado enquadra ao sujeito como vítima, como vivedor, como sujeito de direitos com capacidade de restituir sua condição de sujeito a pesar dos fatos violentos e a situação de marginalidade à que se vê enfrentado com a chegada a um contexto diferente e muitas vezes hostil.

Palavras chave: Deslocamento forçado, construcionismo, vitima sujeito de direto, instituições governamentais e não governamentais vínculos. 


\section{Introducción}

La pretensión de este trabajo radica en la posibilidad de dar a conocer algunos resultados del análisis de la problemática del desplazamiento forzado desde los planteamientos de la Psicología Social Crítica y la Psicología Comunitaria, tratando de evidenciar y visibilizar las implicaciones de las categorizaciones sociales, en este caso la del "desplazado", en el diseño y ejecución de las intervenciones y en la formulación de la política pública. Dicho proceso de investigación, se realizó como tesis para optar al título de Magíster en Psicología Comunitaria de la Universidad de Chile.

Se analiza el fenómeno, no sólo como consecuencia del conflicto armado interno, sino como una estrategia de apropiación de los territorios geoestratégicos de los distintos actores en disputa. De esta manera, se entiende que el desplazamiento forzado en Colombia ha modificado las regiones y han pasado a ser del dominio de los actores armados, ocasionando el despojo principalmente de campesinos, indígenas y población afrocolombiana. Esta mirada, permite ver el fenómeno no sólo desde los impactos psicosociales o desde la visión del daño que causa a las personas individual o socialmente, sino también verlo en la perspectiva de los factores sociales, políticos, económicos que posibilitan el problema y que a su vez lo siguen incrementando y que hace difícil la posibilidad del retorno a los lugares de origen y la prevención del fenómeno mismo. En este sentido, la perspectiva Crítica de la Psicología será adoptada como el horizonte teórico que permitirá analizar el fenómeno en su complejidad y de acuerdo con los actores allí implicados.

La investigación se desarrolló desde una perspectiva cualitativa, ya que ésta permite indagar sobre la complejidad de los procesos de construcción social donde se ven involucrados distintos actores, con intereses particulares. El proyecto está basado en la Teoría Fundamentada de Strauss y Corbin (1997).

El estudio es relevante socialmente por las implicaciones psicosociales, culturales, económicas y políticas que éste plantea a los científicos sociales, y a los actores implicados ya que permite analizar los intereses y los recursos de los que disponen los actores (población desplazada, instituciones gubernamentales y no gubernamentales) para la superación o no de la situación. El propósito de la investigación se orientó hacia la comprensión de la construcción social de la categoría del desplazado desde los actores implicados y los vínculos que construyen con los organismos gubernamentales y no gubernamentales.

\section{Problema}

Con respecto a la grave crisis humanitaria que vive el país, es importante plantear que el Alto Comisionado de las Naciones Unidas para los Refugiados -ACNUR- informó el pasado 17 de junio del año 2008, que Colombia tiene 552.000 refugiados en el exterior, lo que hace que el país se ubique en el tercer lugar después de Afganistán con tres millones y de Irak con dos millones. La situación se hace mucho más grave cuan- 
do se informa que Colombia ocupa el primer lugar con tres millones de desplazados, desterrados y despojados de sus territorios dadas las condiciones del conflicto social y armado que azota al país desde hace más de cuatro décadas. El segundo lugar lo ocupa Irak con dos millones cuatrocientos mil y seguido la República Democrática del Congo con un millón trescientos mil desplazados internos (Santos, M. El Tiempo 2008).

En este sentido, un comunicado de ACNUR (2009) expresa lo siguiente: “...unos tres millones de colombianos se han visto forzados a abandonar sus hogares por razones de violencia, persecución o graves violaciones a sus derechos humanos. Sólo el año pasado, más de 170.000 personas fueron forzadas a huir de sus hogares. Otros cientos de miles han cruzado una frontera huyendo hacia los países vecinos, o aún más lejos."

En el año 2007, CODHES informó que: "un promedio diario de 743 personas fueron desplazadas durante los primeros seis meses del año en flujos migratorios que afectaron 32 departamentos y 579 municipios del territorio nacional. La tendencia se mantuvo en el trimestre siguiente (julio, agosto y septiembre), periodo que coincidió con la campaña electoral para elegir integrantes de cuerpos colegiados y autoridades territoriales."

Con este marco, se puede entender que este fenómeno es de vieja data, pero que es reconocido como tal sólo en la década de los 90, donde se evidencia que los actores armados se disputan los territorios donde habita población civil, por ser territorios geoestratégicos para el desarrollo de sus acciones militares, para los cultivos ilícitos e incluso para la implementación de megaproyectos donde están puestos los intereses de capitales transnacionales. Ello permite problematizar este fenómeno no sólo por la grave crisis humanitaria que se ha generado, sino también porque se ponen en evidencia los intereses de distintos actores no sólo los grupos ilegales, sino también los intereses políticos y económicos que allí se movilizan. Lo anterior, permite reflexionar que difícilmente el Estado colombiano podrá superar esta situación si no se tiene la voluntad real de transformar las causas estructurales que mantienen el problema.

Se entiende que Colombia ha mantenido una guerra interna desde hace más de cuatro décadas que ha tenido expresiones distintas en todas las regiones. En particular en los tres departamentos del Eje Cafetero, específicamente en Risaralda, una de las consecuencias más sentidas que se presenta es la del desplazamiento, fenómeno que incide en el aumento de los cinturones de miseria, empobrecimiento, desempleo en los centros urbanos, en este caso de la ciudad de Pereira, dado que las personas ven en estos lugares una posibilidad de esperanza y centro de oportunidades laborales y sociales. La agudización de esta problemática ha llamado la atención de instituciones gubernamentales y no gubernamentales; en ese sentido, se han dictaminado leyes para atender dicha problemática como la Ley 387 de 1997 compuesta por 34 artículos que dio cabida a la vez a los decretos 951 sobre la Vivienda y el subsidio de Vivienda para la población desplazada del 24 de mayo del 2001, el Decreto 2007 de septiembre 24 de 2001, sobre 
la reubicación y la posibilidad de retorno a las tierras, y el Decreto 2562 de noviembre 27 de 2001 referente a la educación para la población desplazada en etapa escolar.

En la Ley 387 de 1997, se contempla la responsabilidad del Estado con el desplazamiento interno, los principios rectores para su atención, la creación del Sistema Nacional de Atención Integral a la Población Desplazada por la Violencia, (Art. 4 al 8), construcción del Plan Nacional para la Atención Integral a la Población Desplazada, el consejo nacional y consejos municipales para dicha atención. Además de la Ley 387 de 1997, la Corte Constitucional falló la sentencia T-025 de 2004 en la que se exponen los argumentos de la población desplazada en contra de la Red de Solidaridad Social ${ }^{1}$ y otras dependencias gubernamentales por no cumplir de manera adecuada y oportuna la "misión de protección a la población desplazada y por la falta de respuesta efectiva a sus solicitudes en materia de vivienda y acceso a proyectos productivos, atención de salud, educación y ayuda humanitaria." (Sentencia T-025 de 2004). En ese sentido, la Corte determina proteger los derechos constitucionales de la población desplazada y determina que el país se encuentra ante "un verdadero estado de emergencia social, una tragedia nacional, que afecta los destinos de innumerables colombianos y que marcará el futuro del país durante las próximas décadas y un serio peligro para la sociedad política colombiana; y, más recientemente, un estado de cosas inconstitucional.”

De igual manera, la Corte Constitucional expidió el Auto 092 de 2008 donde se plantea la: "Protección de los derechos fundamentales de las mujeres víctimas del desplazamiento forzado por causa del conflicto armado", en el marco de la superación del estado de cosas inconstitucional declarado en la sentencia T-025 de 2004. En el Auto 092 la Corte manifestó: “...adopta medidas comprehensivas para la protección de los derechos fundamentales de las mujeres desplazadas por el conflicto armado en el país y la prevención del impacto de género desproporcionado del conflicto armado y del desplazamiento forzado." (Auto 092 de 2008). Allí se encuentra contenido la creación de 13 programas específicos dirigidos a la población desplazada femenina, generar programas para superar los vacíos en la política pública, el amparo a las mujeres desplazadas en 600 casos específicos y comunicar al Fiscal General de la Nación sobre los crímenes sexuales cometidos en el marco del conflicto armado (Auto 092 de 208).

Las anteriores son evidencias de algunas disposiciones legales y jurídicas que el Estado colombiano ha dictaminado para enfrentar la grave crisis humanitaria que vive el país y que se ha agudizado desde la década de los ochenta.

Por otra parte, se ha podido evidenciar que son múltiples los pronunciamientos donde se manifiesta que este fenómeno ya no es sólo una consecuencia más del conflicto armado. Algunos se atreven a plantear que el desplazamiento es una estrategia de redistribución territorial aprovechada por los actores armados que les permite tener el dominio territorial de sectores estratégicos para el desarrollo de sus actividades mi-

1 Ahora denominada Acción Social de la Presidencia de la República. 
litares (Zuluaga, 2003). Lo otro que es necesario considerar es que según CODHES, "el mapa del desplazamiento forzoso se corresponde con el mapa de formulación o ejecución de macroproyectos en zonas que cuentan con riquezas mineras, energéticas y zonas de cultivos ilícitos y procesamiento de drogas" (Salcedo, 2006). Esto implica entonces problematizar la situación de manera distinta, en el sentido de que los desplazamientos de personas no se dan solamente por las confrontaciones entre actores armados, sino también por la implementación de mega proyectos a desarrollar en zonas estratégicas del país. Lo que ha permitido llegar a la afirmación: "No hay desplazados porque hay guerra, sino que hay desplazados para que haya guerra" (REDIF, 2003).

Por otra parte, defensores de derechos humanos plantean el fenómeno como una violación múltiple a los derechos humanos. Esta frase encabeza buena parte de las bibliografías, noticias, proyectos y estudios que han surgido como respuesta a este fenómeno; aun así, el apoyo a la población desplazada continúa siendo fragmentado, insuficiente, dejando fuera del margen de asistencia varios de los niveles en que son vulnerados: la dignidad de las mujeres, hombres, niñas y niños desplazados.

Cada día el número de personas y familias desplazadas aumenta; pasan los días, meses y años, mientras su situación continúa siendo precaria, se ha invertido tiempo y dinero en promocionar una actitud solidaria frente a las familias desplazadas, comerciales donde se invita a no "invisibilizar" su condición y brindar apoyo, no obstante, estos esfuerzos no han sido encaminados de manera eficiente a las redes de apoyo y a las instituciones que encabezan lo mecanismos de atención a desplazados.

Lo que se evidencia en la mayoría de la literatura que hace referencia al fenómeno en cuestión, plantea la gravedad de la crisis humanitaria por la que pasa el país desde hace unos años dado el recrudecimiento del conflicto social y armado y los impactos que éste ha traído a la población civil, fundamentalmente campesinos, afrocolombianos e indígenas.

En este sentido, vale la pena contribuir a la discusión sobre la dimensión política de este fenómeno. De esta manera, interesa evidenciar cómo se ha construido socialmente la categoría de este sujeto desplazado, conocer sus estrategias de exigibilidad de derechos, implica problematizar la condición de "víctimas" y analizar si es posible otra noción, también como sujetos y actores políticos. Resulta importante evidenciar los mecanismos que se construyen para la exigibilidad de los derechos, del restablecimiento, de la justicia y de la reparación. Pensar al desplazado como actor político, invita a reflexionar la relación entre el Estado, la Sociedad Civil y el desplazado como tal. En esta vía, es importante reconocer que los escasos y débiles procesos organizativos, la naturalización del desplazamiento y la situación de víctimas ayudan a consolidar la imagen de víctima, minimiza la posibilidad de construcción del sujeto desplazado como actor y sujeto político. 
Según las anteriores consideraciones, el problema sobre el que interesa profundizar es sobre la categoría desplazado, qué actores participan, cuáles son las implicaciones para el diseño de las intervenciones y de la política pública y cuáles son los vínculos que éstos generan con los organismos gubernamentales y no gubernamentales, permitiendo construir algunos aportes sobre el tipo de vínculos que se están consolidando entre el Estado y la Sociedad Civil como se mencionó anteriormente.

En este sentido, vale la pena reflexionar si efectivamente las personas en situación de desplazamiento encarnan esta visión de la política. Así, cobra relevancia indagar por la capacidad organizativa de la población en situación de desplazamiento, cuál es su posibilidad para incidir en lo público y en lo político y de esa manera evidenciar si esta capacidad organizativa confluye en procesos de participación política orientada a resolver tanto los problemas particulares como los problemas colectivos que como población desplazada presentan. En esa medida, se hace necesario indagar sobre las relaciones y vínculos que éstos construyen con otros actores gubernamentales y no gubernamentales.

Se entiende que los vínculos que los actores gubernamentales y no gubernamentales establecen con la población desplazada se dan a través de los lineamientos de intervención, sobre todo en el acompañamiento a proyectos productivos y el acompañamiento psicosocial. Lo que interesa entonces son las relaciones entre los desplazados: su capacidad organizativa y de incidir políticamente; con los organismos mencionados. De acuerdo con estos planteamientos, se propone la siguiente pregunta de investigación ¿Cómo se construye socialmente la categoría del desplazado y cuáles son sus implicaciones en los vínculos que se establecen con las instituciones gubernamentales y no gubernamentales? Se propuso como objetivo general comprender la construcción social de la categoría del desplazado desde los actores implicados y los vínculos que establecen con los organismos gubernamentales y no gubernamentales.

\section{Metodología}

Este trabajo investigativo se desarrolló bajo una lógica de la investigación cualitativa, específicamente la Teoría Fundamentada. Para la realización del trabajo de campo se consideraron informantes clave de los actores más relevantes que intervienen en el fenómeno en cuestión. Los narradores fueron identificados de acuerdo con la información otorgada por las instituciones que ya han determinado procesos organizativos: grupos de mujeres, líderes de asambleas barriales y personas en situación de desplazamiento que no pertenecen a ninguna organización. De esta manera, se tuvieron en cuenta los narradores que contaron la experiencia vivida del desplazamiento y los procesos organizativos que lidera o de los que ha participado y se pudieron realizar interpretaciones respecto a cómo se construye la categoría del desplazado desde la perspectiva de los actores intervinientes. 
De igual manera, se hizo necesario abordar a los funcionarios de instituciones gubernamentales y no gubernamentales, asistir a las actividades programadas por la institucionalidad y donde se convocaron a las personas en situación de desplazamiento, con el propósito de observar e identificar las relaciones y vínculos que allí se construyen. Fue necesario un acercamiento a los informes y documentos que han sido producidos por estas instituciones; desde allí se trató de evidenciar las formas discursivas, la manera como se construye la categorización social del desplazado y las implicaciones en los procesos de intervención. Las producciones de los académicos también se consideraron en este interés, se revisaron documentales producidos por la Red Nacional de Investigadores en Desplazamiento Forzado -REDIF-.

Los sujetos se seleccionaron de manera intencionada, dinámica y secuencial. Intencionada, porque se identificaron los actores que cumplieran con las características anteriormente expuestas y no se escogieron al azar. Dinámica, porque la misma dinámica del trabajo de campo permitió identificar estos actores para ser entrevistados. Secuencial porque se hizo uno por uno, respondiendo a la misma dinámica que se fue generando (Patton, 1990).

La técnica empleada fue la entrevista, dado que ésta tiene como objetivo articular un espacio de palabra y de interlocución con aquel que relata su historia y que lleva a considerar ésta, como testimonio irrepetible que superpone a la crónica verídica y cronológica de los acontecimientos del pasado. Queda abierta la puesta en escena de épocas, espacios, situaciones, circunstancias que no son nunca individuales, que son inevitablemente colectivas, sociales y testimoniales. Aquí, se propone profundizar con los actores las situaciones vividas a partir de la experiencia del desplazamiento y profundizar cuáles han sido los mecanismos de organización que ellos y ellas han construido, qué obstáculos, dificultades y aciertos han tenido en dichos procesos. Interesa entonces, reconstruir la historia de las vivencias y conocer la experiencia de quienes han actuado bajo la dirección de las instituciones en los procesos de intervención con la población desplazada. Se espera de esta manera, ahondar en los vínculos que construyen los actores sociales implicados y las posibles implicaciones que ello genera en el diseño de las políticas.

Permitió también identificar qué tipos de relaciones establecen, qué relaciones de poder se generan entre ellos, con otros grupos y con los organismos gubernamentales y no gubernamentales, qué situaciones contradictorias se generan, en fin, encuentros y desencuentros que pudieron aparecer a partir de los discursos que allí se plantean. Además de la entrevista, se realizaron registros de observación y revisión documental.

Por otra parte, se emplearon los tipos de muestreo, los cuales tratan de dar cuenta de los tres tipos de codificación que se proponen desde la Teoría Fundada: muestreo en la codificación abierta, donde la investigadora permita que el interés se dirija hacia muchas situaciones, incidentes o hechos donde puedan aflorar datos interesantes para 
la investigación, aquí la sensibilidad juega un papel importante porque se empiezan a definir cuáles serán los conceptos sobre los cuales se tratará de profundizar para la construcción de las categorías. Posteriormente, se trató de encontrar relaciones que se van construyendo entre las categorías y las subcategorías, con el fin de establecer comparaciones para hallar similitudes o disparidades entre los acontecimientos.

Para el rigor de esta investigación, se emplearon los criterios de suficiencia y adecuación de los datos antes que el número de sujetos entrevistados. La suficiencia se consigue cuando se llega a un estado de "saturación informativa" y la nueva información no aporta elementos nuevos. La adecuación se refiere a la selección de la información de acuerdo con las necesidades teóricas del estudio y el modelo emergente. Se evidencia saturación teórica, cuando no se encuentran datos nuevos para una categoría, éstas se encuentran bien desarrolladas en términos de sus propiedades y dimensiones, se encuentran variaciones y cuando se pueden establecer relaciones suficientes y adecuadas entre las categorías (Strauss, A. \& Corbin, J. 1990, p. 231).

Los tres momentos de dicha reducción de datos se pueden dar simultáneamente; éstos implican la codificación de la información de acuerdo con las categorías de análisis; se utiliza porque los datos son de tipo textual. El criterio por el cual se lleva a cabo la codificación es temático pues ya que la agrupación se hace asociando elementos sobre un mismo tema o categoría.

La metodología, estrategia y técnicas de análisis e interpretación de la información se orientó según la perspectiva de Strauss y Corbin (2002) a partir de lo postulado en la Teoría Fundada.

El procedimiento que se siguió la realización de entrevistas puntuales con funcionarios de instituciones públicas, población en situación de desplazamiento, actores sociales relevantes, revisión de material documental de agencias de cooperación internacional, específicamente la Agencia de Cooperación Técnica Alemana -GTZ- y algunos registros de observación de jornadas de reunión donde se encontraron funcionarios públicos y población desplazada.

Siguiendo los planteamientos metodológicos de la Teoría Fundada, el muestreo teórico permite la "recolección de datos guiada por los conceptos derivados de la teoría que se está construyendo y basada en el concepto de "hacer comparaciones", cuyo propósito es acudir a lugares, personas o acontecimientos que maximicen las oportunidades de descubrir variaciones entre los conceptos y que hagan más densas las categorías en términos de sus propiedades y sus dimensiones” (Strauss, A. \& Corbin, J. 1990, p. 219). En este sentido, se trató de indagar sobre los incidentes, acontecimientos que permitieran darle mayor densidad a las categorías en sus propiedades y dimensiones. Desde esta perspectiva se asume que el muestreo es acumulativo, ya que cada acontecimiento o incidente importante se acumula a lo que el investigador ya ha venido incorporando en sus elaboraciones e interpretaciones. Al principio, el investigador se interesa por ge- 
nerar la mayor cantidad de categorías y posteriormente se dedica a densificar y a saturar las mismas (Strauss, A. \& Corbin, J. 1990, p. 221).

\section{Resultados}

Los resultados se presentarán de manera parcial, por efectos de la extensión del artículo. Se exponen los resultados de cómo se construye la categoría desde el punto de vista de los mismos desplazados y desde las instituciones.

\section{Construcción de la categoría desplazado, desde la perspectiva de los desplazados}

Dentro de esta categoría se evidenciaron tres subcategorías: identidad, estigmatización sobre el desplazado, posibilidad de superar la categoría de desplazados.

Respecto a esta categoría, se identifica que las personas en situación de desplazamiento reconstituyen su identidad alrededor de la figura "desplazado", esta es la categoría que una vez llegados a la ciudad les permite definirse y disponerse ante las instituciones y las organizaciones no gubernamentales: para la exigencia de los subsidios, de los derechos, de la reparación, entre otras cosas: “... a nosotros no nos dieron arriendo, ni ropa, a nosotros aparte del mercado no nos dieron más nada. Y a nosotros nos tenían que dar muchas cosas" (E1P61).

Las personas entrevistadas evidencian que han sido objeto de la estigmatización que hace la población receptora sobre ellos como auxiliadores de la guerrilla, como personas que compiten con los pobres por la atención de las instituciones del Estado, refieren que la población receptora mantiene la creencia que las instituciones privilegian a los desplazados y esto hace que se genere una situación de competencia. La estigmatización que se genera sobre esta población obstaculiza las posibilidades de estabilización laboral y se mantiene un círculo vicioso que hunde a la población en condiciones de pobreza y por lo tanto, se hace más difícil trascender la categorización. "Mientras nosotros seguimos siendo desplazados es un problema conseguir trabajo; la gente dice ¡ah! es que esa desplazada, pero no saben las instituciones que nosotros no somos los guerrilleros, y ellos creen que nosotros por ser los desplazados somos los guerrilleros" (E1P101).

Además de lo anterior, se demuestra que estas personas mantienen una conveniencia al autodefinirse como "desplazados", a pesar del tiempo que ha transcurrido (más de ocho años del desplazamiento en las personas que fueron entrevistadas) se siguen presentando y definiendo como desplazados, planteando que todavía no han obtenido todas las "ayudas" y el restablecimiento del Estado para superar la condición del desplazamiento. Es reiterativo en las entrevistas y en el discurso de la población desplazada que el gobierno o las instituciones "les dan o no las ayudas" como si se tratara de 
favores recibidos o no, aunque algunos reconocen que no se trata de ayudas o favores sino que más bien se trata de la responsabilidad que el Estado tiene con ellos, "Yo sufrí mucho, es una experiencia muy dura, y luego el otro hijo que estaba en la policía me llevó pa' todo lo que era Cundinamarca, pero eso me dio muy duro, allá me pusieron un psicólogo, y así después de eso ya como al año fue que yo ya volví y declaré y entonces ya salieron las ayudas del gobierno" (E1P25). "(...) pero como aquí en Pereira a nosotros no nos han cumplido con esas cosas entonces seguimos ahí desplazados" (E1P96).

Es importante expresar que algunas de las personas desplazadas, hacen alusión a la necesidad de superar su condición del desplazamiento, expresando que "tenemos que sacarnos esa idea de la cabeza, nosotros no toda la vida vamos a ser desplazados" (E1P102). Se hace explícita la posibilidad de trascender la condición de desplazamiento pero se enuncia también que "como a nosotros no nos han dado lo que nos pertenece, seguimos siendo desplazados" (E1P103). Se mantiene una especie de contradicción entre la necesidad de trascender la condición y su mantenimiento porque aún no han sido reparados en su totalidad y mientras esto no suceda, el Estado deberá seguir atendiendo sus necesidades.

\section{Vínculos con instituciones}

Respecto a esta categoría se evidencian vínculos con instituciones gubernamentales y con Organizaciones No Gubernamentales.

En las entrevistas y observaciones realizadas de actividades donde se encontraron funcionarios públicos y población desplazada, se generaron situaciones de tensión, de reclamos e incluso de acusaciones. Los líderes manifiestan que aún no han sido atendidas todas sus necesidades, que han recibido malos tratos de los funcionarios y hacen acusaciones a algunas ONG operadoras de Acción Social porque éstas no entregaron todos los recursos a los que tenían derecho para la generación de proyectos productivos. En el siguiente planteamiento, se evidencia desconfianza de las organizaciones de desplazados hacia algunas entidades porque no conocen cuáles han sido los manejos y la administración que se ha hecho sobre los recursos con que deben ser atendidas sus necesidades. "¿Entonces cuál es la relación que tienen con Acción Social? La directora de allá; a nosotros no nos dieron arriendo, ni ropa, a nosotros aparte del mercado no nos dieron más nada. Y a nosotros nos tenían que dar muchas cosas, y entonces ella viene ahorita y los de Acción Social le están haciendo el seguimiento" (E1P71).

Sólo una de las mujeres entrevistadas relató haber sido adecuadamente atendida por los funcionarios de la Personería Municipal y de la Unidad de Atención y Orientación al desplazado. Llama la atención que esta mujer expone desempeñarse en la actualidad como manipuladora de alimentos en el programa de seguridad alimen- 
taria que desarrolla la Alcaldía, además manifiesta haber trabajado en la campaña electoral del actual alcalde y que es bien recibida por la primera dama, incluso que ésta privilegia atenderla sobre otras personas en su misma condición y por ello se han generado algunos conflictos.

En algunas ocasiones se hace evidente que los candidatos aprovechan las campañas electorales para generar promesas a la población vulnerable (entre ellas la población desplazada), generándose un mecanismo de favores por votos que se traduce en clientelismo. En esta medida, la población desplazada reconoce que el principal interés es poder resolver las necesidades y entienden que para lograrlo se requiere recurrir a este tipo de mecanismos.

Las organizaciones de desplazados generan vínculos con entidades estatales a través de las demandas y acciones de tutela que interponen para que sus derechos sean reconocidos; ellos manifiestan que todo lo que han logrado ha sido mediante la vía de la tensión y el conflicto con las entidades, ya que perciben que éstos no reconocen plenamente sus derechos. "Ahora estamos peleando, luchando para que el alcalde haga el favor de pavimentar la vía. Porque es que esto después de que lo pavimenten queda con otra cara. Entonces el alcalde ya se comprometió con eso, se comprometió en el colegio para pavimentar esto, y la vía de acá a la universidad”(E1P101P102).

Lo anterior, ha generado relaciones de desconfianza entre las entidades estatales y la población desplazada y ha permitido que las tensiones se hayan profundizado en la mayoría de las ocasiones. Los desplazados perciben que no han recibido todo a lo que ellos tienen derecho, llegando a hacerse innegable un reclamo de atención de tipo asistencial. Por otra parte, sienten que los funcionarios los ven a ellos como peligrosos, conflictivos, dependientes y mentirosos porque se les entregan los subsidios y ellos los siguen reclamando. A pesar de que algunos desplazados reclaman subsidios, "ayudas", otros de ellos manifiestan la necesidad de superar esta relación de dependencia y asistencial, ya que son ellos mismos los que deben mejorar sus condiciones de vida. Al respecto se manifiesta que: "Eso es un problema para todos, nosotros no podemos seguir siendo a diario desplazados no. A nosotras donde nos hubieran dado ya los proyectos productivos y todo lo que nos tienen que dar, no estuviéramos con esa cosa de desplazados, ya no, pero como a nosotros no nos han dado lo que es que nos pertenece tener seguimos siendo desplazados”(E1P101P102).

Respecto a las entidades no gubernamentales, los desplazados hacen manifiesta su desconfianza, critican que en muchas ocasiones se han generado promesas que no se han cumplido y que además éstas reciben grandes cantidades de recursos para la generación de proyectos productivos que no han sido adecuadamente utilizados y entregados. Algunos de ellos hacen acusaciones porque sus proyectos productivos fueron rechazados y porque no saben cuál fue el destino de estos recursos. En este caso, llama la atención que estas entidades no realizan rendición de cuentas a las comunidades de 
desplazados, mecanismo que podría ayudar a restablecer los niveles de confianza si las personas conocieran en qué y cómo se han invertido estos recursos.

Estas personas relatan cómo fueron atendidas en la fase de emergencia, realizando toda la ruta de atención: desde la declaración en la UAO o Defensoría del Pueblo, la aceptación de su condición y la posterior entrega de los subsidios de arriendo y alimentación. Aún así, siendo beneficiarios de estos aspectos, ellos exponen que esto ha sido y sigue siendo insuficiente, porque los subsidios que les entregan son escasos, no alcanzan para cubrir las necesidades de toda la familia, que en la mayoría de las veces son bastante numerosas (familias de cuatro o más hijos). A partir de esto, empieza entonces todo un proceso de exigencia a las entidades gubernamentales y no gubernamentales con el propósito de obtener mayores recursos y que éstos apunten a la implementación de la segunda fase: la de restablecimiento económico. En este aspecto, se manifiesta que algunas entidades no gubernamentales no han entregado todos los recursos que éstas han recibido por las agencias de cooperación internacional.

Manifiestan además que su deseo es poder superar el carácter asistencial que se mantiene en estas fases de la intervención y que imposibilita un proceso de mayor autonomía que se traduzca en la cesación de la condición de desplazamiento. En este aspecto, llama la atención que la población manifiesta que éste es su deseo pero contradictoriamente es repetitivo el planteamiento de que es el gobierno el que debe seguir ofreciendo lo que ellos denominan "las ayudas".

\section{Construcción de la categoría desplazado desde la perspectiva de las instituciones}

El desplazado como víctima. Desde el punto de vista de los psicólogos que trabajan para una institución no gubernamental, se aprecia que el desplazado es visto de distintas maneras; en esta vía, se puede trabajar la idea de que la figura del desplazado no es una esencia, una cosa que se pueda conocer y desentrañar, sino más bien que se trata de una categoría que ha sido construida socialmente por los distintos actores que se encuentran allí involucrados (Gergen, 2003). Desde una de las perspectivas, los desplazados son vistos como "víctimas", son las personas que han tenido que migrar de manera forzada de sus lugares de origen, despojados de bienes, asustados, temerosos en búsqueda de una "ayuda" en la ciudad. Lo anterior, que corresponde a la descripción de estos sujetos en la fase de emergencia, parece perpetuarse a través del tiempo y la victimización parece asumirse como una característica más de su propia identidad; es una especie de cosificación de la condición de víctima, que muchas veces no se quiere o no conviene abandonar porque permite obtener los beneficios económicos. Pero por el hecho de que tienen más beneficios, tienen más ayudas si se quedan ahí. Es como si conviniera no trascender la condición de desplazado porque así se siguen recibiendo los beneficios y por lo tanto, se evidencia en algunos casos que no hay un interés real para superar esta condición. 
Un caso concreto narrado por uno de los psicólogos lo describe así: "un hombre que lleva más de ocho o diez años acá en la ciudad, y yo lo he visto más o menos en tres años, todo lo que él ha hablado y no ha cambiado su discurso, sigue diciendo lo mismo que es la víctima y que el Estado lo tiene que proteger" (E3P115).

Un aspecto que es llamativo para estos profesionales es que las instituciones mismas se encargan de victimizar la población desplazada "los vuelven más víctimas"; entonces como "yo soy la víctima, yo tengo derechos, y todo para mí." Aquí las instituciones mismas tienen una responsabilidad en la configuración de estas víctimas, generando una relación de dependencia con las instituciones. Se menciona que cuando se pretende problematizar este asunto desde el proceso de acompañamiento psicosocial, se les pide a algunos que hagan un esfuerzo y ante esto responden: "no, es que a mí me tienen que ayudar. Se convierte en una persona demandante, que succiona los procesos" (E3P125). Cuando se le atribuye al otro la condición de víctima se le quita la posibilidad de considerarlo como actor de su propia transformación.

\section{Estigmatización del desplazado.}

Se identifica la estigmatización que recae sobre la población desplazada, las comunidades receptoras consideran que ellos son intrusos o que generan algún peligro porque han pertenecido a algunos de los actores armados, situación que dificulta mucho más la integración social e incluso impide que esta población pueda acceder a oportunidades laborales estables y duraderas. "Llega como el malo del pueblo, el que perteneció a grupos armados pero se salió y lo encontraron”(E3P19).

\section{El desplazado como objeto de intervención}

Sobre el desplazado recae el estigma de víctima, y ha sido convertido también en objeto o destinatario de la política pública. Sobre estos sujetos se han diseñado leyes, decretos, sentencias, autos y demás recursos legales y jurídicos que han puesto la atención sobre esta población para intervenir su condición. Se analiza que durante estos diez años de emergencia de la política pública, los desplazados han sido destinatarios de la aplicación de estos lineamientos y de los programas y proyectos de intervención propuestos por las instituciones gubernamentales, no gubernamentales y las agencias de cooperación internacional. No obstante, sólo recientemente esta población empieza a ser considerada como sujetos con capacidad para incidir y participar en la toma de decisiones, incluso empiezan a ser consideradas las organizaciones de desplazados para participar en el diseño y consolidación de esta política pública. De todas maneras, aún se sigue quedando en la intención y hace falta avanzar mucho más en la consolidación de los procesos organizativos y su capacidad de incidencia y participación en los escenarios donde se diseñan las políticas que trazarán los mecanismos de intervención. 88 Se esperaría que en un futuro próximo el desplazado no sea "objeto" sino "sujeto" de 
la intervención, el acompañamiento, la reparación y el restablecimiento en todas sus dimensiones.

\section{El desplazado como sujeto de derechos y agente de transformación}

La Corte Constitucional y la Ley asumen que el desplazado es un sujeto de condiciones especiales de protección; por su parte, la población desplazada expone que ellos deben conocer muy bien sus derechos para "protegerse de alguna manera del Estado, o exigirle al Estado” (E3P12). Algunos conocen sus derechos, otros no y otros a medias, pero en la medida que pasa el tiempo y deben acercarse a las instituciones para las reclamaciones de los subsidios, de la ayuda humanitaria y otros, éstos van asumiendo un rol más desde la exigibilidad de sus derechos y algunos se ocupan de asistir y participar de todas las jornadas de capacitación que deben ofrecer las instituciones para profundizar sus conocimientos y por lo tanto, hacer más efectiva la exigibilidad de sus derechos. También se hace necesario el reconocimiento no sólo de los derechos, sino también de los deberes y responsabilidades de la población desplazada. Los psicólogos hacen mucho énfasis en esto, ya que encuentran que la población desplazada es muy dada a exigir sus derechos pero a conocer poco sus deberes.

\section{Superación de la categoría desplazado}

A pesar de que algunos mantienen la condición de víctimas por los elementos anteriormente expuestos, se encuentran personas que plantean cómo esta condición debe ser superada a partir de sus propios esfuerzos y compromisos. Se reconoce la necesidad de organizarse como población desplazada, que las instituciones tienen su responsabilidad pero que ellos también se tienen que organizar y aportar desde su posición para trascender esta situación. Algunos casos ratifican el planteamiento de que es posible encontrar personas que vivieron el desplazamiento pero que ya han superado esta condición. Lo anterior, permite además superar las relaciones de dependencia que se han construido con las instituciones.

La condición de víctima y de desplazado se asume como algo que hay que superar y dejar en el pasado; se confronta a los compañeros que atraviesan esta condición y que mantienen una queja constante de su situación de desplazado, se les cuestiona porque ésta debe ser superada y dejada en el pasado. Un caso ilustrativo de lo anterior evidencia que hay algunas personas que efectivamente expresan que esta condición se puede superar: "Tengo otro conocido que perteneció a la JUCO en los años ochenta, lo torturaron, eso fue pues una cosa compleja, acá lo volvieron a desplazar, yo tuve que intervenir en crisis, fue la primera persona que yo vi en crisis y él ahorita me saluda y yo le pregunto cómo va y me dice, no, eso ya pasó, yo ya no tengo nada que ver estoy muy bien" (E3P309). 
Los psicólogos exponen que vale la pena aclarar que la mayoría de estos casos que conocen, son personas que han contado con redes de apoyo y factores de protección que les ha permitido superar la situación; al respecto manifiestan que: "claro las condiciones son muy distintas; por ejemplo, estos dos señores tenían familiares y conocían ya la ciudad, sabían donde ir y cómo hay que hablar sin problema. Entonces son como factores de protección que pueden ayudar a que ellos piensen en eso. Por ejemplo, tengo la radiografía de un indígena, a él lo desplazaron del Putumayo y ya sabía manejar el computador; está estudiando para ser profesor y dice que el desplazamiento es muy difícil pero que ya no es la víctima, 'soy igual que usted', dice” (E3P313).

\section{Conclusiones}

Se reconoce que los conceptos y categorías también son representaciones mediadas por la forma de su apreciación y percepción; es decir, por la perspectiva metodológica y epistemológica que instituye al científico; pero no son sólo representaciones de ese objeto, son también las prácticas que dichas representaciones performan y provocan el advenimiento de la realidad social. Es importante dejar claro que las categorizaciones son de "manejo público" del colectivo que los elabora, y que se establecen y se fijan vía socialización, etc. Las construcciones categoriales que se hacen pueden provocar el advenimiento de la realidad, esto es que la realidad la asumimos como la representamos, este es el carácter performativo de los conceptos y categorías sociales, y de los que pretenden objetivar realidades sociales.

De esta manera, sobre el desplazado recaen, no sólo las nominaciones que se hacen sobre el mismo, si es víctima, aprovechado, actor/agente o acomodado, éste incorpora no sólo lo que se dice de él, sino que actúa como se espera que actúe. Aunque lo anterior también es susceptible de interpelación, ya que esto no se da así de una vez y para siempre, es posible trascender dicha situación; afortunadamente queda algo del sujeto que escapa de las determinaciones, de las categorizaciones. Este escape incluso le permite incluirse como sujeto en un contexto que ha sido excluyente con él. Lo que habría que analizar es cómo ha sido posible este escape o este proceso de inclusión, con qué mecanismos cuenta, de qué se dotaron esos sujetos, en qué pugnas se tuvieron que involucrar, qué tuvieron que ganar y qué tuvieron que perder. A veces las cosas aquí se tratan de ganar y perder, se gana o se pierde reconocimiento, se gana o se pierde poder para incidir, para mantener o transformar las condiciones que parecen imponerse, muchas veces por la fuerza.

Vale la pena entonces reconocer, que este "sujeto desplazado" no puede ser visto como esencia de la realidad social, sustancializar la misma es lo que impide desnaturalizar aquello que ha sido naturalizado. Si algo se le abona a la teoría social crítica, es precisamente su capacidad para desencializar lo que parece natural (Ibáñez, 1994. Piper, I. 2002). Pues bien, este sujeto, muchas veces liminal y marginal, no es per se liminal, es 
porque los actores sociales con los cuales se las tiene que ver, han permitido que esto sea de esta manera, y no se trata de victimizar a la víctima, se trata de reconocer también que éste responde a los juegos del lenguaje y lo que ello genera; él juega también a ser desplazado para obtener lo que considera "las ayudas", para sobrevivir o para exigir lo que le pertenece: las tierras o para exigir que se le reconozcan sus derechos. De esta manera se dispone y en esa disposición entra incluso en tensiones y pugnas con otras categorías sociales, los considerados como los "pobres históricos" y muchas veces, le va mejor a alguien que dice ser desplazado que pobre. No se quiere decir con esto que toda categoría conceptual exista por el derecho de autoridad de quien la enuncia, ni mucho menos que al existir y tomar fuerza la categoría produzca la existencia de lo que enuncia o categoriza. Insistimos en que se trata de modelos de representación que resultan ser perfomativos porque al necesitar de ellos para orientarnos en la realidad, estamos construyendo la realidad de tal manera que estos nos puedan orientar en ella; independientemente de su naturaleza que si bien puede ser teórica (para el científico) o social -o del sentido común- (para el sujeto corriente), resultan ser modelos desde cualquier perspectiva.

Respecto a la organización como población desplazada, les va mejor si están organizados; en esta medida, se hace necesario reconocer que para el caso de los sujetos entrevistados (actores institucionales y desplazados), los segundos se organizan porque de esta manera pueden acceder de manera más expedita a los recursos de las instituciones estatales o de las agencias de cooperación internacional. De ahí que no sea gratuito que estas entidades, desde hace un buen tiempo vienen planteando en sus planes de intervención, la necesidad de trabajar el fortalecimiento organizativo de la población, en el propósito de que el desplazamiento sea una condición susceptible de ser trascendida, abandonada. Aquí valdría la pena preguntarse sobre las posibilidades reales de la población de retornar a sus tierras, ya que definitivamente el desplazamiento forzado se ha convertido en una estrategia de guerra para la apropiación de los territorios estratégicos para los actores armados, incluso para el mismo Estado colombiano y las empresas transnacionales que tienen sus intereses económicos puestos en tales territorios. Así, la reclamación de la tierra aparece como uno de los temas más álgidos del fenómeno, tema que nadie quiere tocar; si se les pregunta a los funcionarios, éstos responden aludiendo a los decretos, los mandatos de la Corte Constitucional y demás; no obstante, los funcionarios no responden de manera clara y contundente y mucho menos exponen sobre las posibilidades reales del retorno con garantías.

$\mathrm{Si}$ tratásemos de responder a la pregunta ¿Qué significa ser un desplazado en Colombia? Tendría que responderse que se trata de una categoría que ha sido construida socialmente, con la participación y el concurso de los distintos actores que tienen competencia directa con el fenómeno; en esta vía, se puede entender que el desplazado no es desplazado en sí mismo, sino que éste se convierte en tal por unas condiciones objetivas que se han generado en el país: el conflicto, el desarrollo y dinámicas del 
mismo, que ha provocado que hayan personas que tengan que emigrar de sus lugares de origen por las condiciones de peligro inminente contra su vida o la de sus familias; pero no basta con saber que hay unas condiciones objetivas, sino que tiene que ver también con la manera cómo se construye, cómo se ve, cómo se percibe y cómo se trata ese sujeto hombre o mujer desplazado, tiene que ver con cómo institucionalmente o desde los actores se consolida una idea o una categoría de lo que es el desplazado. Y éste ha sido edificado como un sujeto liminal, marginal al que hay que mantenerle unas condiciones, que como lo plantean las mismas instituciones gubernamentales, "de ayuda", cuestión que requiere ser problematizada y porque no se trata de una ayuda sino de la responsabilidad que tiene el Estado respecto al mantenimiento de un conflicto y de su incapacidad para resolver los problemas que han mantenido por más de cuatro décadas el mismo. Además, de la responsabilidad que éste tiene para facilitar, provocar, promover las capacidades en la población mediante los recursos técnicos, humanos y financieros para modificar lo que ha sido llamado por la Corte como un estado de cosas inconstitucional que atenta contra las condiciones de vida digna que deben ser defendidas en el Estado Social de Derecho.

Respecto al fenómeno del desplazamiento, se pudo evidenciar que los actores indagados dan cuenta de que este fenómeno obedece al escalonamiento del conflicto armado con las distintas dinámicas que éste puede tener en las regiones, pero además se encuentra que los actores no estatales reconocen que el desplazamiento forzado se ha intensificado durante los últimos años por dos razones básicamente: la implementación de la política de seguridad democrática del actual gobierno de Álvaro Uribe Vélez y por la implementación de megaproyectos en regiones de carácter geoestratégico. De esta manera, se pudo evidenciar que los actores que participan en la construcción social de la categoría, son actores gubernamentales que actúan desde el nivel nacional hasta el nivel local. Las instancias del nivel nacional como Acción Social junto con los ministerios se encargan de dar las directrices que deben ser cumplidas por las instancias del nivel local, las que la mayoría de las veces carecen de recursos humanos, técnicos y financieros para darle cumplimiento. Por otra parte, se pudo evidenciar que la Corte Constitucional ha desempeñado un papel importante en el proceso de reconocimiento de los derechos de la población desplazada, así como también ha permitido que las instancias nacionales e internacionales tengan la responsabilidad de reconocer la población no sólo en su condición de víctima sino también en su condición de agentes con capacidad para organizarse y realizar la exigibilidad de sus derechos.

Otros actores de gran relevancia lo han constituido las agencias de cooperación internacional, las cuales con el apoyo técnico y financiero han facilitado procesos de intervención con la población desplazada; se reconoce que algunas de estas agencias han empezado a hacer mayor énfasis en la generación de capacidades y no exclusivamente en la ayuda humanitaria o la intervención de tipo asistencial. Se pudo reconocer también que la academia ha venido generando y circulando un discurso impor- 
tante respecto al fenómeno del desplazamiento, no sólo por los constantes análisis que desde allí se hacen sobre el desarrollo del conflicto social y armado del país, sino porque un grupo de académicos de importantes universidades han decidido apostar a la construcción del debate sobre la situación dramática del desplazamiento forzado, llegando a constituir la Red Nacional de Investigadores en Desplazamiento Forzado -REDIF-, cuyo propósito inicial fue recoger los trabajos de investigación provenientes de distintas disciplinas y profesiones que dan cuenta del fenómeno en las distintas regiones del país. Al momento, REDIF realiza un encuentro anual donde se socializan dichos trabajos y cuentan con más de diez publicaciones referidas al desplazamiento, además de la cátedra permanente que se imparte en asocio con el Alto Comisionado de Naciones Unidas para los Refugiados -ACNUR-y la Universidad Nacional de Colombia, en las universidades de todo el país que decidan impartir la cátedra a sus estudiantes como una manera de sensibilizar a los futuros profesionales y de generar debate y discusión sobre las nuevas dinámicas del conflicto armado y el desplazamiento forzado.

Se consideró también la necesidad de conocer las discusiones generadas desde la Sociedad Civil respecto al fenómeno del desplazamiento en Pereira; por ello, se pudo realizar una entrevista con el Consejero Departamental de Paz (instancia que agrupa asociaciones y organizaciones que velan por el cumplimiento de los derechos humanos en la región), quien es conocedor de la dinámica del conflicto en Pereira y la región y ha generado mecanismos de exigibilidad de verdad, justicia y reparación de algunos casos puntuales de desplazamiento ante instancias gubernamentales del contexto departamental y nacional.

Por obvias razones, también se identificó la población desplazada como un actor central en la construcción social de esta categoría, se quiso evidenciar cómo éstos asumen e incorporan la condición de ser desplazado en un contexto que la mayoría de las veces es excluyente, con la precariedad de la intervención estatal y con las implicaciones de la victimización y el asistencialismo.

Desde el punto de vista de las instancias gubernamentales mediante la Guía de Atención Integral a la Población Desplazada por la Violencia en Colombia (2007), el Estado colombiano reconoce la magnitud y complejidad del fenómeno del desplazamiento y la vulneración de derechos que de allí se derivan y propone. "realizar el mayor esfuerzo para atender a la población afectada por el desplazamiento forzado, un esfuerzo en el cual concurren diferentes entidades públicas y privadas, organismos de cooperación internacional y organizaciones no gubernamentales, comunitarias y de la sociedad civil, con el propósito de mejorar la capacidad de respuesta frente al desplazamiento y de generar alternativas que permitan superarlo" (GAIPDVC, 2007. pp. 7-8).

Es decir que Colombia es reconocida a internacionalmente como el país donde se producen los mayores esfuerzos para atender la población desplazada afectada por el 
desarrollo del conflicto armado. Además, se reconoce que aquí confluyen los esfuerzos de las entidades públicas, privadas, de la cooperación internacional, las Organizaciones No Gubernamentales y la Sociedad Civil con el propósito común de mejorar la capacidad de respuesta para atender las necesidades de la población desplazada.

Hasta el año 2002, la dinámica del desplazamiento forzado había mantenido una tendencia al aumento y afirman que una vez implementada la política de seguridad democrática del actual gobierno de Álvaro Uribe Vélez esta dinámica empezó a decrecer (GAIPDVC, 2007. p.18) Los anteriores datos, se evidencian como contradictorios con lo encontrado en las entrevistas a actores sociales no gubernamentales, los académicos, el consejero departamental de paz, los movimientos sociales, quienes afirman que es a partir de la implementación de la política de seguridad democrática cuando el fenómeno del desplazamiento se agudiza y en consecuencia hay una tendencia al aumento.

De lo anterior, se desprende que en Colombia no hay consenso entre las agencias, entidades y organizaciones no gubernamentales sobre el aumento o no de la población desplazada. Al respecto la Consultoría para los Derechos Humanos (CODHES) expone que en el país hay cerca de tres millones de personas en situación de desplazamiento, las entidades gubernamentales exponen que hay 2.169.874 personas desplazadas hasta el 3 de septiembre de 2007. Más allá de la exactitud de los datos, interesa visibilizar las contradicciones emergentes desde las distintas posturas asumidas por los agentes sociales que necesariamente conllevan a tensiones entre estos actores en la búsqueda por el reconocimiento de sus intereses y sus respectivas implicaciones para la construcción de las políticas de atención del fenómeno.

Por otra parte, desde el punto de vista de las Organizaciones No Gubernamentales que han actuado como operadores de programas y proyectos de intervención financiados con recursos de las entidades gubernamentales y de las agencias de cooperación internacional, se puede concluir que:

El gobierno nacional, departamental y local realiza los mínimos esfuerzos en recursos humanos, técnicos y financieros para atender las necesidades de la población desplazada.

Las intervenciones se quedan en el nivel asistencial y en la atención de la fase de emergencia, realizando esfuerzos mínimos para satisfacer las necesidades básicas de esta población, además que no se reconocen las características diferenciales de los distintos grupos poblacionales afectados, además que no se reconocen las necesidades reales de la población.

Las intervenciones de tipo asistencial dejan de lado aspectos subjetivos como los deseos y expectativas de la población.

De estas intervenciones de emergencia y de tipo asistencial, se generan y mantienen relaciones de dependencia. Por lo anterior, las instituciones y la población desplazada mantienen una lógica paternalista que no permite la superación de la condición de víctima. 
En las instituciones prevalece el interés de cumplir con los indicadores; es decir, que interesa más presentar los informes a las instancias de control con indicadores satisfactorios, más no el interés principal de mejorar las condiciones de vida de esta población.

En el desarrollo de los programas y proyectos se generan relaciones de hostilidad con las instituciones públicas, privadas y con la población.

Con lo anterior, cada vez se hace más recurrente el discurso de los funcionarios, quienes plantean que la Sentencia T-025 de la Corte, declara el estado de cosas inconstitucional que vio una violación masiva, sistemática y continua de los derechos de la población desplazada y también se evidenciaron unos problemas de la política de atención a la población desplazada con la asignación insuficiente de recursos y la incapacidad institucional; todo esto se vio reflejado en el Auto 008 que dice que a pesar de que el gobierno ha estado presentando informes a la Corte Constitucional sobre algunos avances en algunas áreas importante de la política pública, no se ha demostrado suficientemente que sí se haya superado el estado de cosas inconstitucional, ni que se esté avanzando de forma acelerada y sostenida hacia su superación, porque el Estado tiene que estar presentando paulatinamente informes a la Corte y ellos van analizando si realmente ha habido avances y qué logros se han obtenido hacia la superación del estado de cosas inconstitucional y en el cumplimiento del goce efectivo de los derechos. En este aspecto, la Corte ha sido enfática en plantear que la situación de desplazamiento mantiene un estado de cosas inconstitucional y que las instituciones no han generado una política pertinente y eficaz para superar tal estado de cosas.

Por lo anterior, la Corte se concentra en algunos aspectos principalmente: la adopción y aplicación de indicadores de goce efectivos; es decir, que las instancias gubernamentales deben presentar un conjunto de indicadores en todos los temas para realizar la medición sobre el cumplimiento o no de todos los derechos de la población desplazada: los indicadores de vivienda, de salud, de educación, de participación, de reunificación familiar, de estabilización socioeconómica, entre otros. Este conjunto de indicadores socioeconómicos ya fue presentado a la Corte y sobre éstos, se empezará a hacer el seguimiento y la medición del cumplimiento de las diferentes instituciones en el tema de la política pública.

Asimismo, la Corte Constitucional es concluyente con los siguientes aspectos: 1) Se evidencia un bajo compromiso de las entidades territoriales, puesto que se demuestra que el tema del desplazamiento no es un tema prioritario en la agenda política de las instancias gubernamentales del nivel local. 2) Se evidencia insuficiencia de recursos humanos, técnicos y financieros para atender la gravedad de la situación de desplazamiento. 3) Insuficiencia en la coordinación de esfuerzos presupuestales de las entidades territoriales; es decir, que no se encuentran en los diferentes municipios y gobernaciones unos presupuestos relativamente acordes a la situación que tiene cada departamento; en algunos se encontró que tienen un presupuesto muy bajo comparativamente a la 
problemática que tienen el departamento y el municipio. 4) Precariedad de la capacidad institucional nacional para efectuar seguimiento, adoptar correctivos y comparar los resultados alcanzados por las diversas entidades territoriales. 5) Falta seguimiento del Ministerio del Interior y de Justicia y de las otras entidades del orden nacional en lo que tiene que ver con la aplicación de la política en el territorio: municipios y departamentos. 6) Disparidades en las entidades territoriales en cuanto a la evaluación de la situación del desplazamiento forzado y la correspondiente respuesta.

Se puede concluir que con la investigación se aportaron algunos aspectos para la comprensión de esta categoría, los vínculos que se construyen entre los actores y las implicaciones a que ello conlleva en el diseño, planeación y ejecución de la política pública de atención del fenómeno del desplazamiento. Al respecto, se puede exponer que uno de los aspectos a considerar es que la política pública aún dista de aportar en la consolidación de los procesos de participación y organización de la población desplazada. Hace falta avanzar mucho más en acortar la brecha entre la formalidad de la jurisprudencia y el cumplimiento efectivo para la población afectada, ya que existen obstáculos para cumplir cabal y eficazmente la política.

Se asume que dependiendo de cómo se moldee y se represente la categoría del desplazado, se sentarán las bases para la intervención del fenómeno. En este sentido, el desplazado ha sido visto como: sujeto especial de derechos, desterrado/despojado, víctima/liminal/marginal, peligroso/colaborador de actores armados, estorbo, vividor/ aprovechado, agente/sujeto de derechos y deberes. 


\section{Referencias}

Bello, M. (2001). Desplazamiento Forzado y Reconstrucción de Identidad. Santa fe de Bogotá: ICFES.

Blanco, A. y De La Corte, L. (2003). "Introducción a la Perspectiva de Martín-Baró, Prologo . En Martín-Baro, Poder, Ideología y Violencia. Madrid: Editorial Trotta.

Castaño, B. (1998). Violencia Política y Trabajo Psicosocial. Bogotá: Fundación Avre.

Cerullo, R. y Weisenfeld, E. (2001). "La concientización en el trabajo psicosocial comunitario desde la perspectiva de sus actores”. En Revista de Psicología, año/vol. X, número 002. Universidad de Chile.

Consultoría para los Derechos Humanos y el desplazamiento (2007). Codhes informa. Boletín de prensa. Número 72, Bogotá, Colombia, 30 de noviembre de 2007.

González, A. (2007). "La población desplazada en Colombia: de la construcción de un perfil como actor colectivo a los desafíos de la organización. Consideraciones teórico-metodológicas para su abordaje". En Restablecimiento, reparación y procesos organizativos de la población en situación de desplazamiento. Publicación de la Red Nacional de Investigadores en Desplazamiento Forzado - REDIF-

Fernández, R. (2006). Investigación cualitativa y psicología social crítica en el Chile actual: Conocimientos situados y acción politica. Forum Qualitative Sozialforschung /Forum: Qualitative Social Research [On-line Journal], Vol. 7 (4), Art 38. Disponible en: http://www.qualitative-research. net/fqstexte/4-06/06-4-38-s.htm

Flick, U. (2004). “Codificación y categorización”. En Introducción a la investigación cualitativa (192212). Madrid: Editorial Morata (Cap. XV).

Gergen, K. (2003). "Realidades y Relaciones. Aproximaciones a la construcción social.” Paidos Básica. Guia de Atención Integral a la Población Desplazada por la Violencia en Colombia, Actualización, 2008.

Palacio, J. y Sabatier, Colette (2002). "El Contexto en Colombia. La Violencia Política: Presiones sobre el concepto". En Impacto Psicológico de la Violencia en Colombia. Salud mental y redes sociales en familias desplazadas en el Caribe. Cap. 2 (5-67) Barranquilla: Ediciones Uninorte.

CODHES (2001). Informe Sistemas de Información y Cifras sobre el Desplazamiento Forzado en Colombia. Informe. Bogotá: CODHES.

Incalcaterra, A. (2004). "El ejercicio de la ciudadanía y el derecho a tener derechos de los miembros de la población desplazada". En Memorias del Seminario Nacional de Derecho de Ciudadanía y Población en Situación de Desplazamiento. Bogotá, 28 de octubre de 2004.

Jaramillo, J. (2007). "La política pública sobre atención a población desplazada en Colombia. Emergencia, constitución y crisis de un campo de prácticas discursivas”. En Tabula Rasa, enero-junio, No. 006. p. 309-338. Bogotá, Colombia.

MPP (Medios para la Paz) (s.f.). Personas en situación de desplazamiento forzado. Sección apoyada por la Agencia Internacional para el Desarrollo (USAID). En: http://www.mediosparalapaz.org

República de Colombia. Ley 387 de 1997.

República de Colombia. Corte Constitucional. Sentencia T-025 de 2004. Magistrado ponente: Manuel José Cepeda. Bogotá. 
República de Colombia. Corte Constitucional. Auto 092 de 2008. Magistrado ponente: Manuel José Cepeda. Bogotá.

Sánchez, A. (2002). "Dispositivos de empoderamiento para el desarrollo psicosocial”. En Universitas Psychologica, julio-diciembre, año/vol. 1 número 002 Pontificia Universidad Javeriana. Bogotá, Colombia.

Strauss, A. \& Corbin, J. (2002). "El muestreo teórico". En Bases de la investigación cualitativa. Técnicas y procedimientos para desarrollar la teoría fundamentada. (219-235). Medellín: Editorial Universidad de Antioquia.

Tovar, C. (2006). "Desplazamiento forzado y acompañamiento psicosocial: a propósito de la emergencia de nuevos actores políticos". En Universitas Psychologica, enero-abril, año/Vol. 5, número 001. Pontificia Universidad Javeriana. Bogotá, Colombia.

Uprimny, R. (2004). "Justicia Constitucional y desplazamiento forzado en Colombia”. En Memorias del Seminario Nacional de Derecho de Ciudadania y Población en Situación de Desplazamiento, Bogotá, 28 de octubre de 2004.

Valles, M. (1997). Diseños y Estrategias Metodológicas. Técnicas Cualitativas de Investigación Social. Reflexión Metodológica y Práctica Profesional. Madrid: Editorial Síntesis.

Zuluaga, J. (2001) “Antecedentes y Tendencias del Desplazamiento forzoso en Colombia”. En: El desplazamiento por la violencia en Colombia Experiencias, análisis y posibles estrategias de atención en el Departamento de Antioquia. Medellín: ACNUR. http://www.un.org/spanish/News/fullstorynews.asp?newsID=8854\&criteria1 $=$ Colombia. 16 de julio de 2008. 01:15 a.m. 